\title{
Research of Resonator Fabrication Technology Based on Silicon Resonant Pressure Sensor
}

\author{
Yide Peng ${ }^{1,}$, Zhengyuan Zhang $^{2, b}$ and Chunhai Zhang ${ }^{1, c}$ \\ ${ }^{1}$ Chongqing University of Posts and Telecommunications, Chongqing, China \\ ${ }^{2}$ National Laboratory of Analog Integrated Circuits, The twenty-four institute of Electronics \\ Technology Group Company, Chongqing, China \\ a1259559219@qq.com, ${ }^{b}$ zhangzy456789@163.com, ${ }^{\mathrm{c}}$ 813633748@qq.com
}

\begin{abstract}
Keywords: Resonant pressure sensor, Resonator, Reactive ion etching, wet chemical etching, Self-etch-stop etching.

Abstract. The research of beam resonator fabrication technology has been done by using reactive ion etching, wet chemical etching and self-etch-stop etching. The difficulties of hollowing out resonator have been solved. The butterfly-shaped beam resonator of resonant pressure sensor is obtained, the other measurement are under the way.
\end{abstract}

\section{Introduction}

Micromechanical pressure sensors have been widely used in industries such as automotive electronics, household appliances, robots and aerospace. Traditional pressure sensor does not have high accuracy and long term stability until resonant pressure sensor came out. Resonant pressure sensor measures pressure by measuring the resonant frequency of the resonator. This paper does a research on fabrication for the resonator of resonant pressure sensor, designs the steps for fabrication resonator and obtains a butterfly-shaped beam resonator successfully by using reactive ion etching, wet chemical etching and self-etch-stop etching. All of these works make a foundation of the fabrication for the whole silicon resonant pressure sensor.

\section{Structure of Beam Resonator}

The structure of the beam resonator is shown in Fig. 1, beam resonator is etched in a silicon chip and is restrained by four ligatures which attach them to the two supporting piers. Under the piers is the diaphragm, pressure under this diaphragm made piers move upwards and apart, increasing the tension of ligatures, cause the resonant frequency change of the resonator. This resonant pressure sensor use electrical excitation to make resonator oscillate. The oscillation makes the clearance between beam resonator and detecting electrode change. Then, the capacitance between them changes. By measure the capacitance changes, the resonant frequency of the resonator is also known. Both of the driving and detecting electrodes are consist of metal films on another oxidized silicon chip, which is bonded to the first chip. It is a very rough task to etch a deep groove under resonator to hollow out the chip. To solve this problem, this paper use reactive ion etching, wet chemical etching and self-etch-stop etching to design a fabrication process and obtain a butterfly-shaped resonator. 


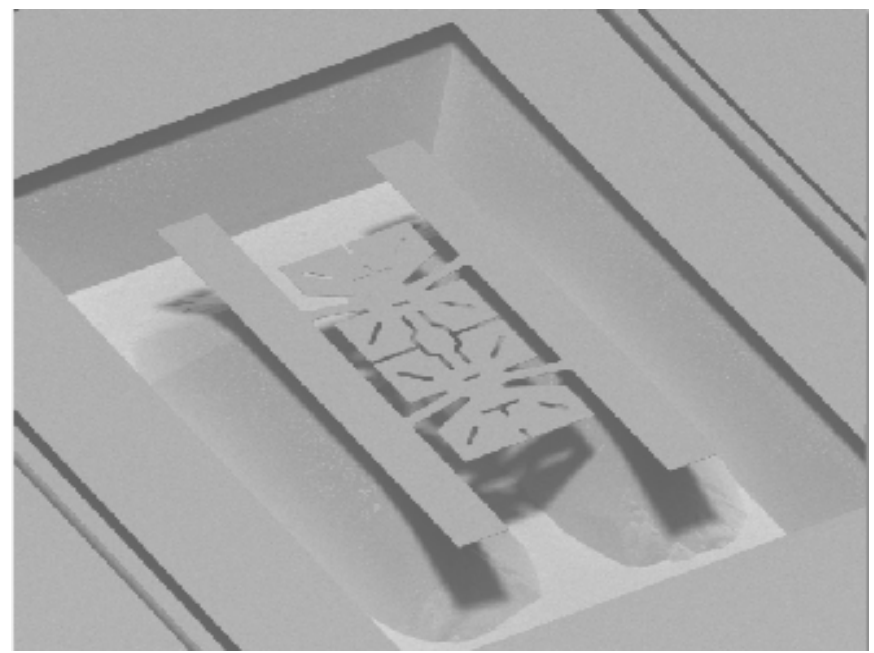

Fig. 1 Structure of resonator

\section{Key Techniques of Resonator Fabrication}

Reactive Ion Etching (RIE). During the process of fabrication, we use RIE to etch $\mathrm{Si}_{3} \mathrm{~N}_{4}$ to form the photomask of wet etching and etch $S i$ exposed by the mask to make wet etching more effective. The difficulties of this step are how to reduce the polymer remained after etching and the ion damage. After our study, we found that we can reduce the polymer by adjusting reaction chamber pressure or radio-frequency (RF) power and send some $\mathrm{O}_{2}$ or $\mathrm{Ar}$ to etching gas at the same time. Increasing reaction chamber pressure, reducing RF power and increasing gas flux can reduce ion damage. As a result, we can get a idol photomask by controlling these parameters.

Wet Chemical Etching. We use $\mathrm{KOH}$ solution to etch silicon chip. The shape of beam resonator we designed is shown in Fig. 2. Slotting at the resonator can make good use of anisotropic wet etching so that the chip could be hollowed. It can also reduce the damping when beam osillating, which improvs quality factor. The concentration of $\mathrm{KOH}$ solution as well as temperature should be low so that self-etch-stop etching could achieve. Because this etch reaction would produce $\mathrm{H}_{2}$ and produce a lot of bubbles, which may cause the reaction slowdown and the surface become rough, we need to stir while etching so that we can make $\mathrm{H}_{2}$ emitted.

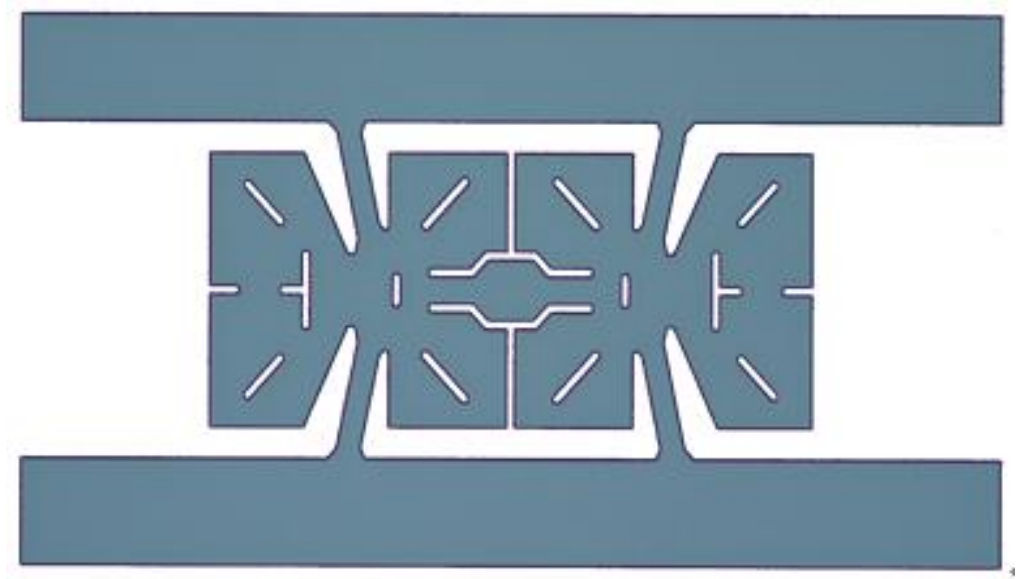

Fig. 2 Shape design of beam resonator

Self-etch-stop Etching. This technique utilize that etchant has different speed of etching for silicon of different doping density. When doping $B$ into silicon, there is a threshold concentration, if the density beyond this concentration, the speed of etching fell sharply. So before RIE, we need to do this doping step first. Then when etching this silicon chip, the beam resonator would not etch. Using ion implantation can control the depth and doping density accurately. 


\section{Fabrication of Resonantor}

Based on our research, we design the steps to fabricate beam resonator:

P (100) oriented silicon wafer -- Silicon wafer polishing on both sides -- Ion implantation on both sides - Annealing -- Oxidation on both sides - LPCVD $\mathrm{Si}_{3} \mathrm{~N}_{4}$ on both sides -- Front photolithography - RIE $\mathrm{Si}_{3} \mathrm{~N}_{4}-\mathrm{RIE} \mathrm{SiO}_{2}$ - RIE $\mathrm{Si}$-- Stripping of photoresist - $\mathrm{KOH}$ wet etching - Etching $\mathrm{Si}_{3} \mathrm{~N}_{4}-$ Etching $\mathrm{SiO}_{2}$-- Wafer rinse.

Finally we obtain a beam resonator as shown in Fig. 3.

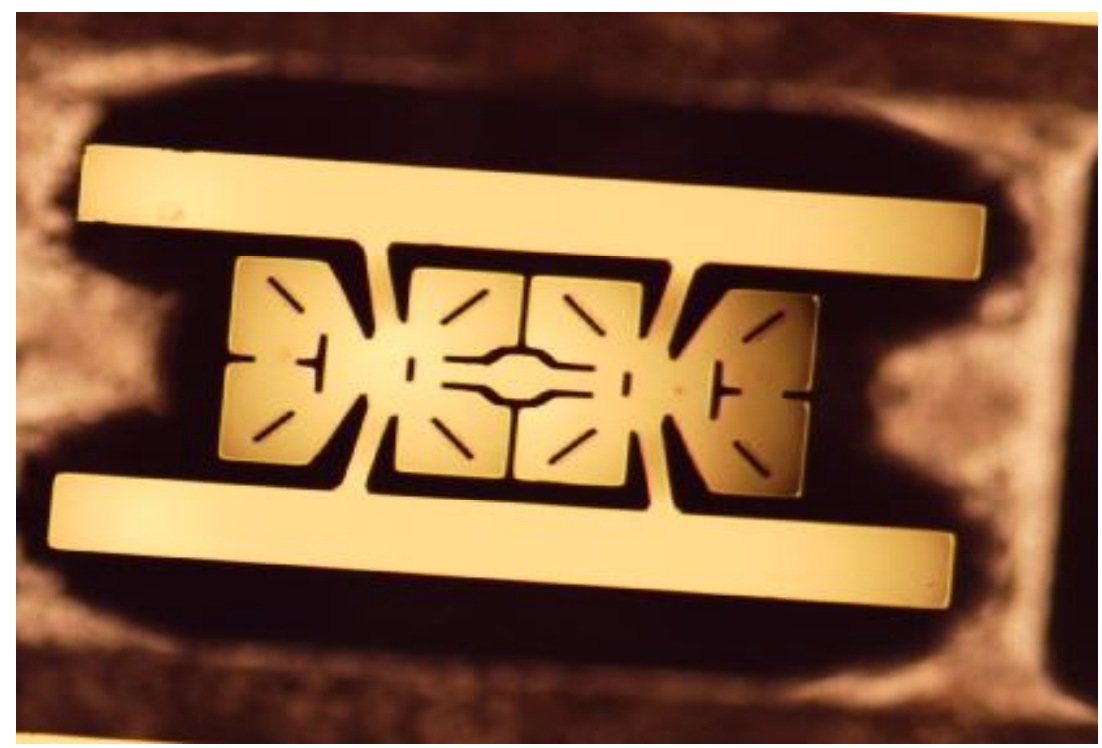

Fig. 3 Beam resonator we obtained

\section{Conclusions}

To ensure the beam is fully released, we take a profile picture of wafer .As shown in Fig. 4, silicon under the center of the beam isn't fully etched. After analysis, we think the effect of etch could be better if we RIE deeper. So we adjust the depth of RIE from $30 \mu m$ to $50 \mu \mathrm{m}$ and take a profile picture as shown in Fig. 5, the problem has solved.

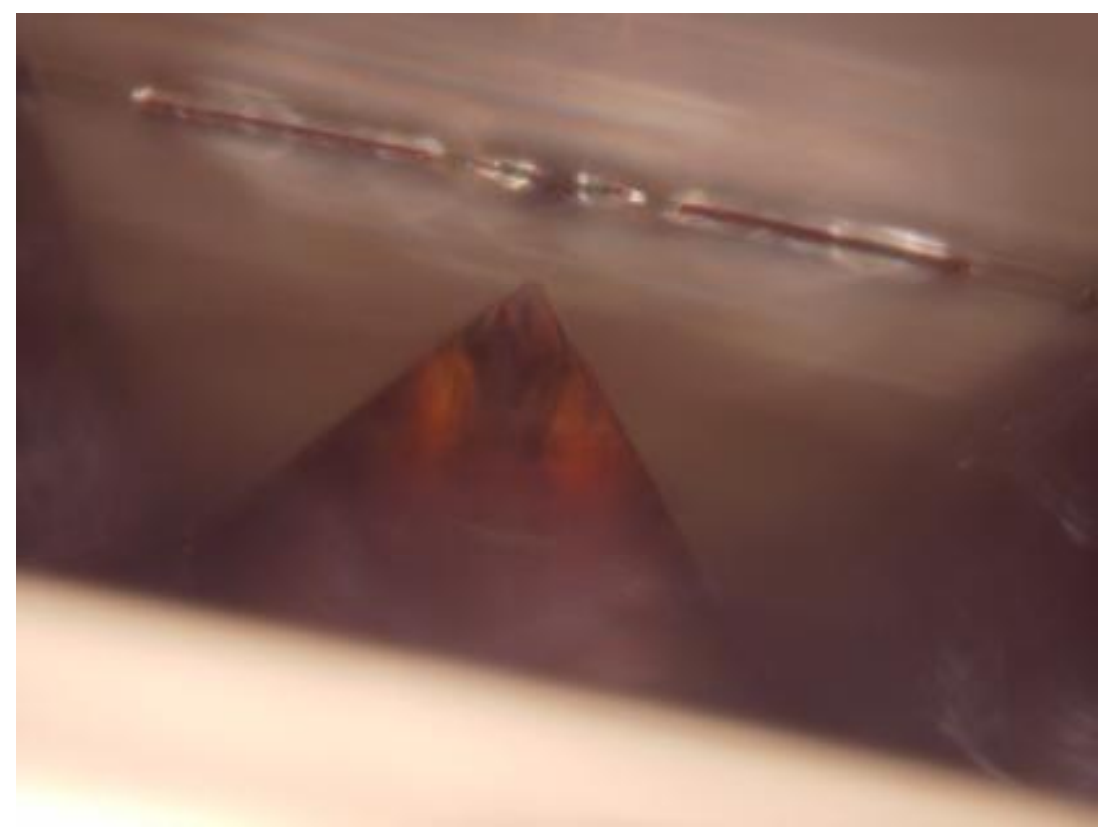

Fig. 4 Profile picture of resonator 


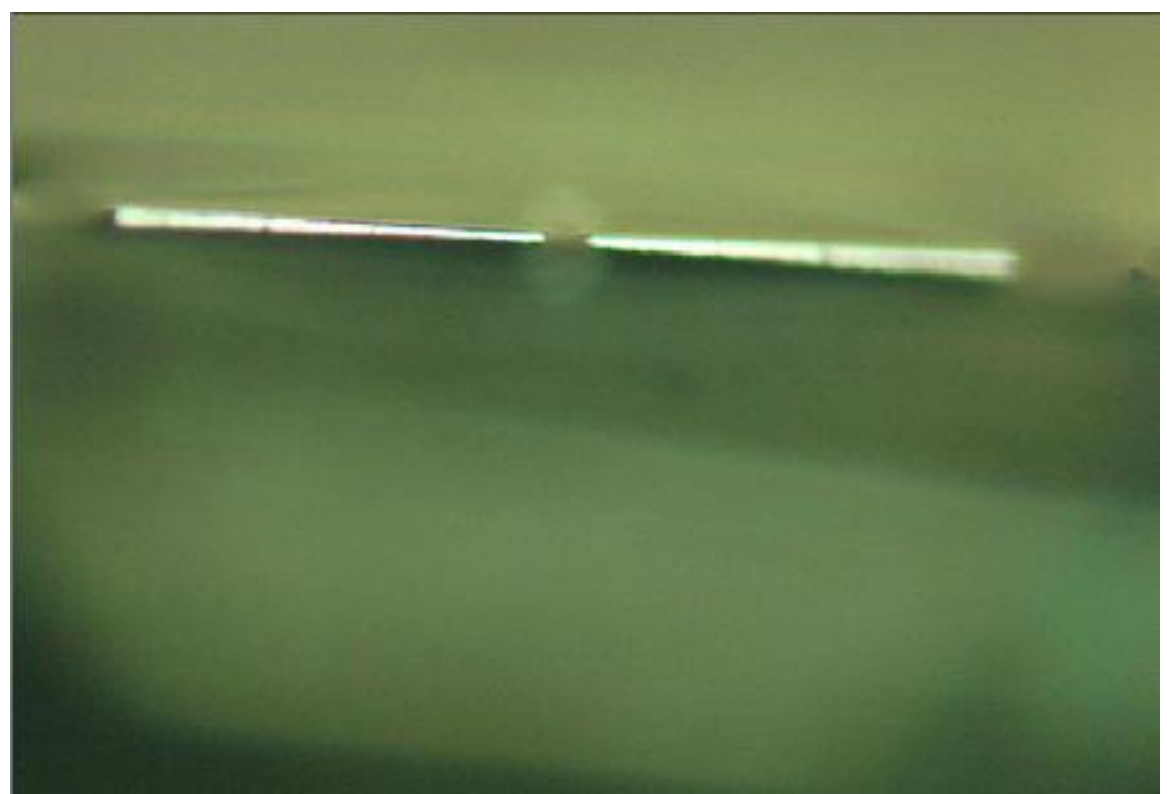

Fig. 5 Profile picture of resonator after improvement

\section{References}

[1] Michael C. Harvey: Design and Fabrication of Micro-Electro-Mechanical Structures for Tunable Micro-Optical Devices. Air University U.S. (2002)

[2] Jordi Teva Merono: Integration of CMOS-MEMS Resonators for Radiofrequency Applications in the VHF and UHF Band. (2007)

[3] K. Hiller, M. Küchler, M. Dienel: T. Gessner. The Bonding and Deep RIE (BDRIE) technology approach for high aspect ratio sensors and actuators. (2005)

[4] M. Elwenspoek.:Mechanical Microsensors. (2001)

[5] Sen Ren: Design and Fabrication of Micromechanical Silicon Resonant Sensor. Northwestern Polytechnical University. (2007) 